\title{
Elevating silicon into a high performance nonlinear optical platform through the integration of 2D graphene oxide thin films
}

David Moss ( $\sim$ dmoss@swin.edu.au )

Swinburne University of Technology

\section{Research Article}

Keywords: 2D materials, silicon photonics, graphene oxide, Kerr nonlinearity

Posted Date: May 10th, 2021

DOl: https://doi.org/10.21203/rs.3.rs-511259/v1

License: (c) (1) This work is licensed under a Creative Commons Attribution 4.0 International License.

Read Full License 


\section{Abstract}

Layered two-dimensional (2D) GO films are integrated with silicon-on-insulator (SOI) nanowire waveguides to experimentally demonstrate an enhanced Kerr nonlinearity, observed through self-phase modulation (SPM). The GO films are integrated with SOI nanowires using a large-area, transfer-free, layerby-layer coating method that yields precise control of the film thickness. The film placement and coating length are controlled by opening windows in the silica cladding of the SOI nanowires. Owing to the strong mode overlap between the SOI nanowires and the highly nonlinear GO films, the Kerr nonlinearity of the hybrid waveguides is significantly enhanced. Detailed SPM measurements using picosecond optical pulses show significant spectral broadening enhancement for SOI nanowires coated with 2.2-mm-long films of $1-3$ layers of GO, and 0.4-mm-long films with 5-20 layers of GO. By fitting the experimental results with theory, the dependence of GO's $n_{2}$ on layer number and pulse energy is obtained, showing interesting physical insights and trends of the layered $\mathrm{GO}$ films from 2D monolayers to quasi bulk-like behavior. Finally, we show that by coating SOI nanowires with GO films the effective nonlinear parameter of SOI nanowires is increased 16 fold, with the effective nonlinear figure of merit (FOM) increasing by about 20 times to $F O M>5$. These results reveal the strong potential of using layered GO films to improve the Kerr nonlinear optical performance of silicon photonic devices.

\section{Full Text}

This preprint is available for download as a PDF.

\section{Figures}




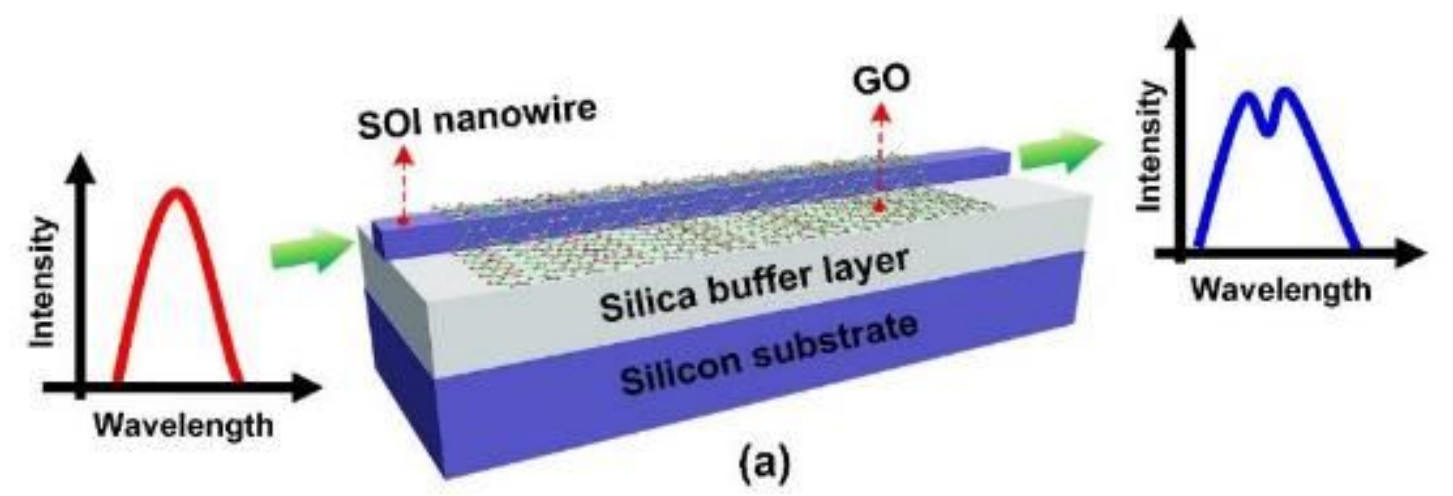

(i) SOl wafer

(ii) Waveguide patterning

(iii) Cladding deposition $\&$ window opening

(iv) GO deposition
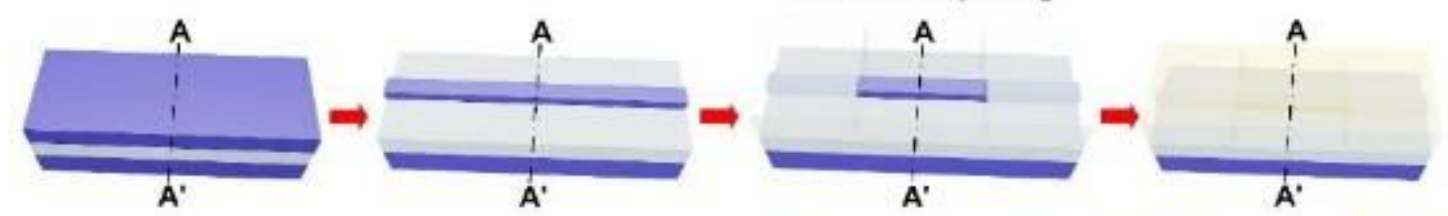

$A-A^{\prime}$

A - $\mathbf{A}^{\prime}$

$\Rightarrow$
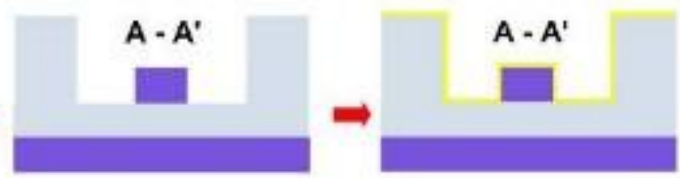

(b)

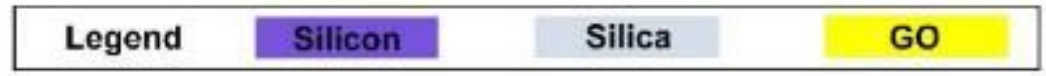

Figure 1

(a) Schematic illustration of a GO-coated SOI nanowire waveguide. (b) Schematic illustration showing the fabrication process flow. 


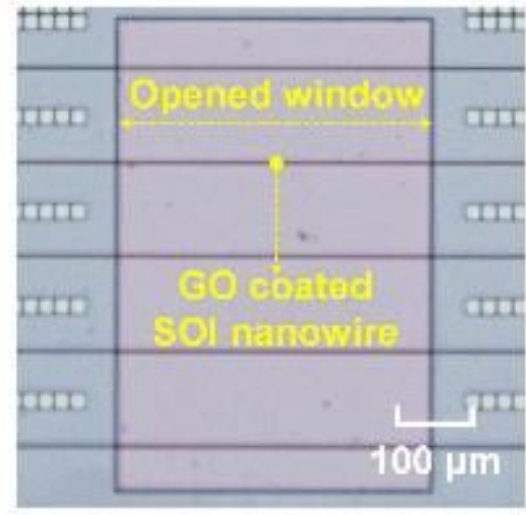

(a)

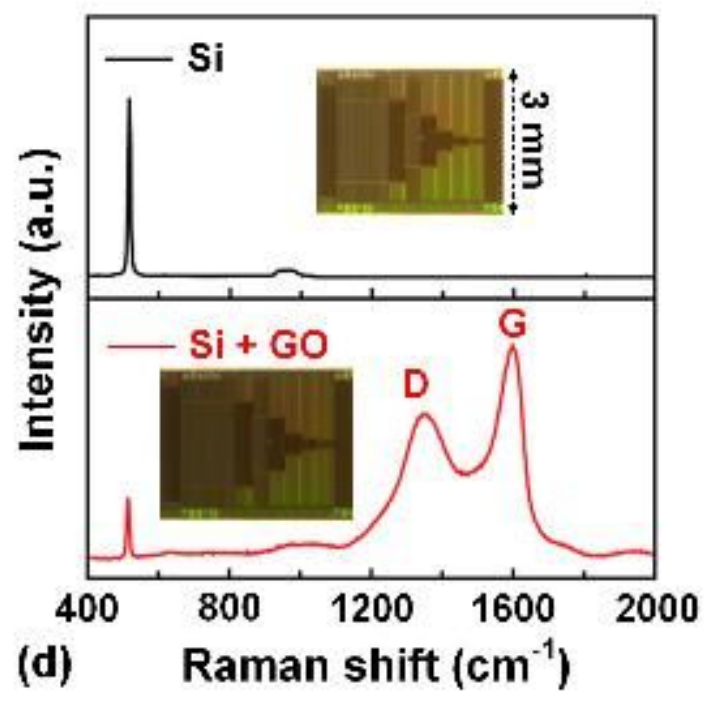

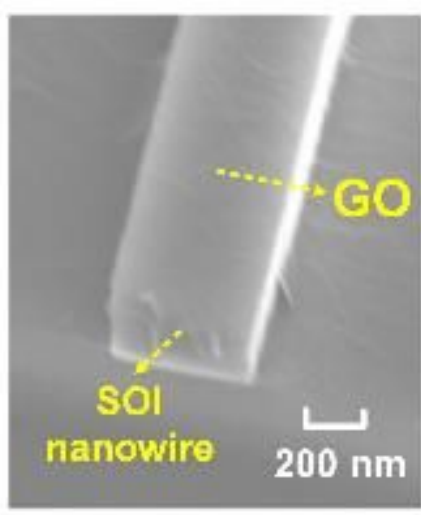

(b)

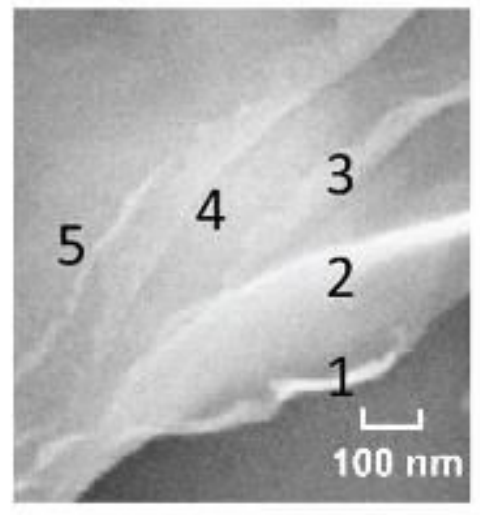

(c)

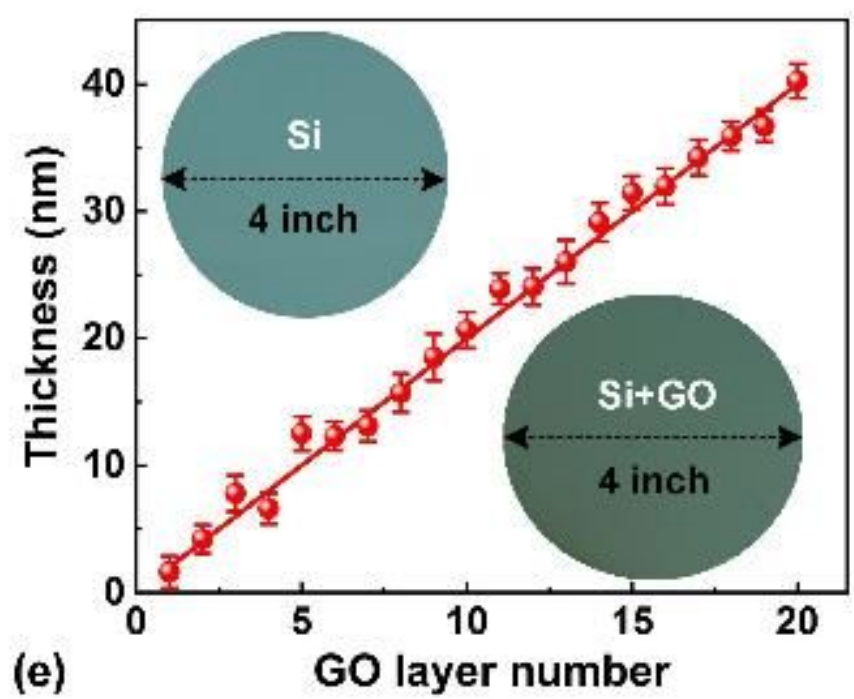

Figure 2

(a) Microscope image of a fabricated SOI chip with a 0.4-mm-long opened window. (b) Scanning electron microscopy (SEM) image of a SOI nanowire conformally coated with 1 layer of GO. (c) SEM image of layered GO film. The numbers refer to the number of layers for that part of the image. (d) Raman spectra of an SOI chip without GO and with 5 layers of GO. Insets show the corresponding microscope images.

(e) Measured GO film thickness versus GO layer number. The plots show the average of measurements on three samples and the error bars reflect the variations. Insets show the images of an uncoated silicon wafer and the same wafer uniformly coated with 20 layers of GO. 

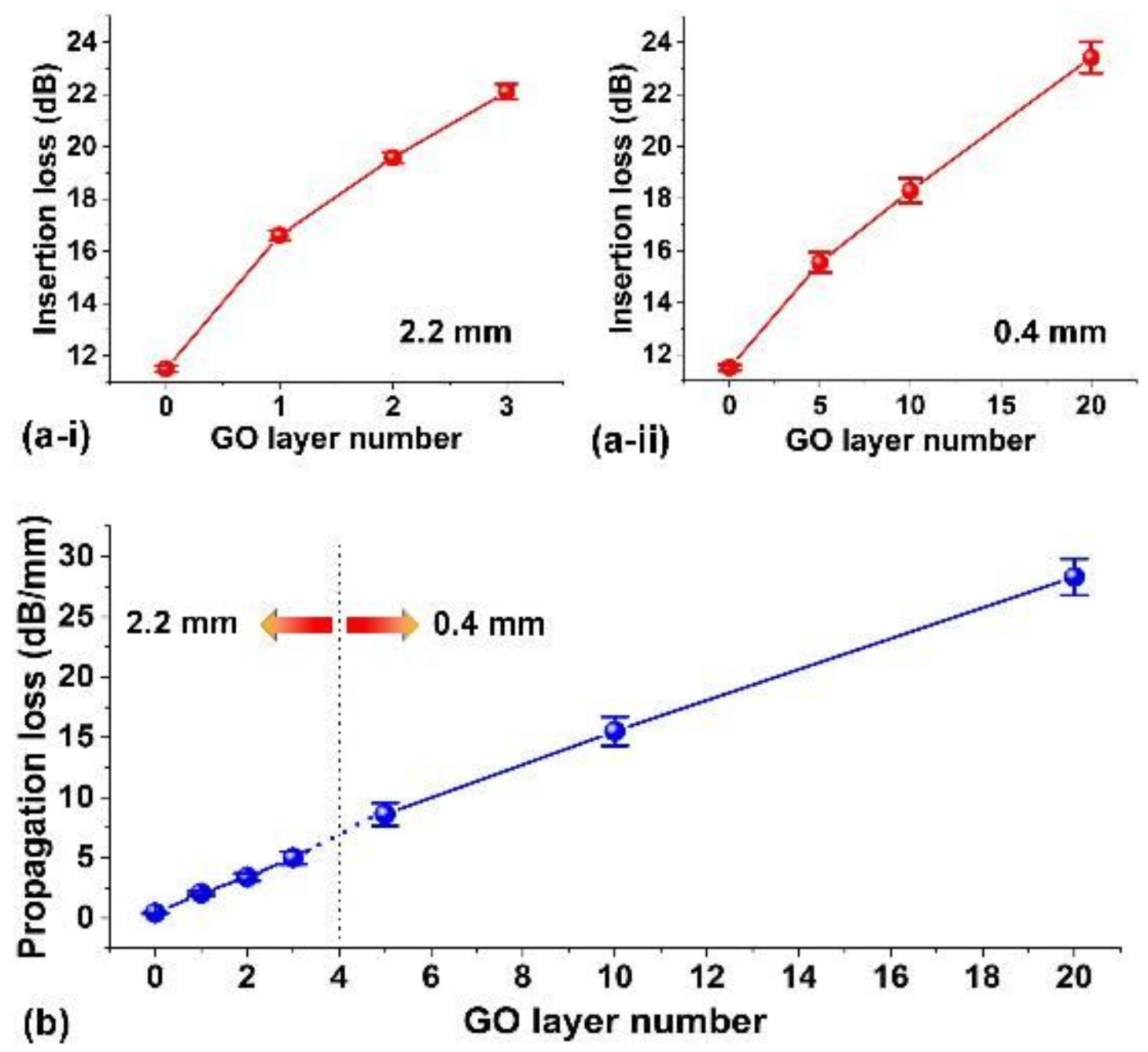

Figure 3

Linear loss measurement. (a) Measured insertion loss of SOI nanowires with (i) 2.2-mm-long, 1-3 layers of GO and (ii) 0.4-mm-long, 5-20 layers of GO. (b) Propagation loss of the GO-coated SOI nanowires extracted from (a-i) and (a-ii). In (a) and (b), the results for the bare SOI nanowires (i.e., the GO layer number is 0 ) are also shown for comparison. The data points depict the average values obtained from the measured insertion loss of three duplicate devices and the error bars illustrate the variations among the different samples. 

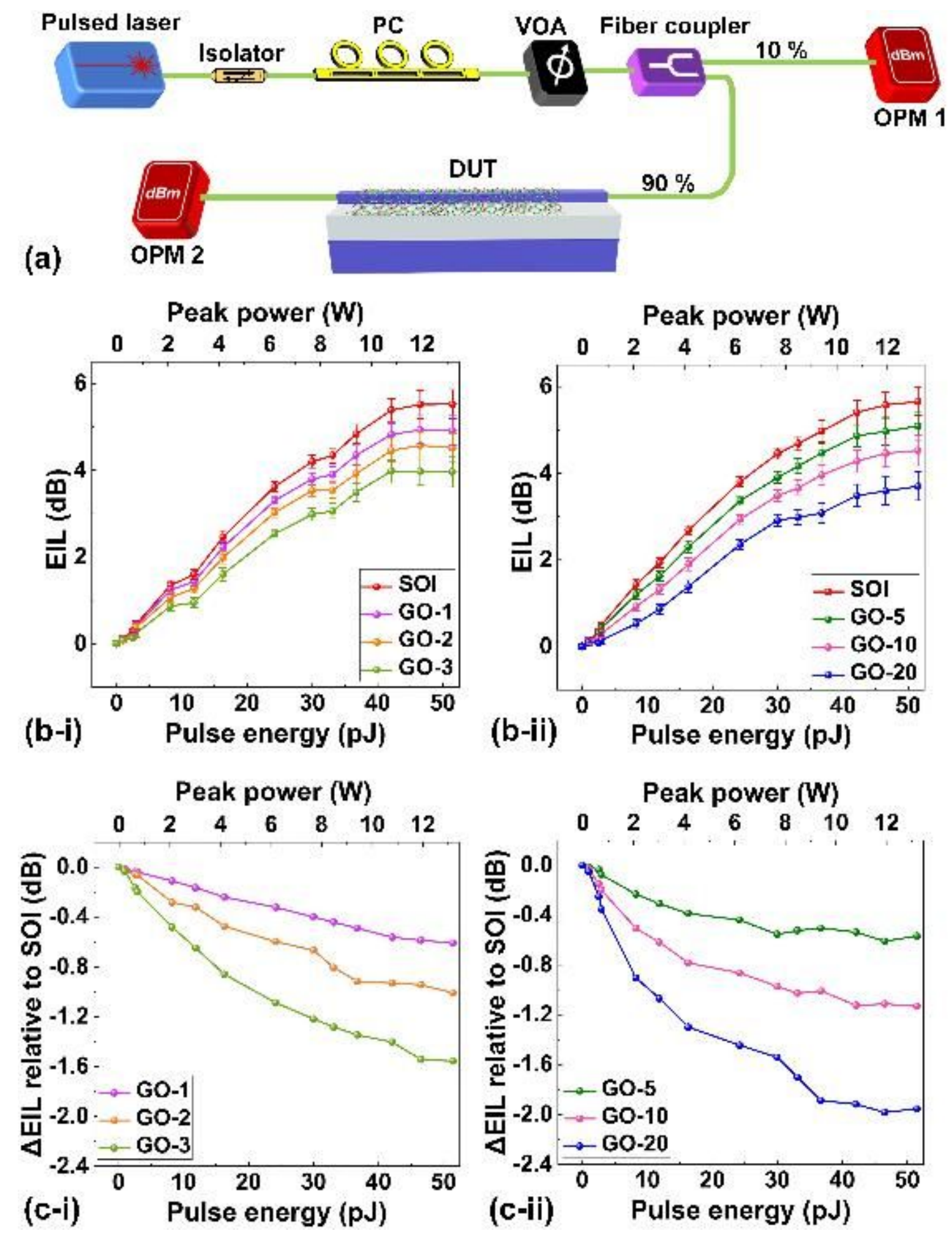

\section{Figure 4}

Nonlinear loss measurement. (a) Experimental setup for measuring nonlinear loss of the GO-coated SOI nanowires. PC: polarization controller. VOA: variable optical attenuator. OPM: optical power meter. DUT: device under test. (b) Power-dependent excess insertion loss (EIL) of optical pulses after transmission through SOI nanowires with (i) 2.2-mm-long, 1-3 layers of GO and (ii) 0.4-mm-long, 5-20 layers of GO. The results for the bare SOI nanowires (SOI) are also shown for comparison. (c) $\Delta$ EIL relative to SOI extracted from (b). 


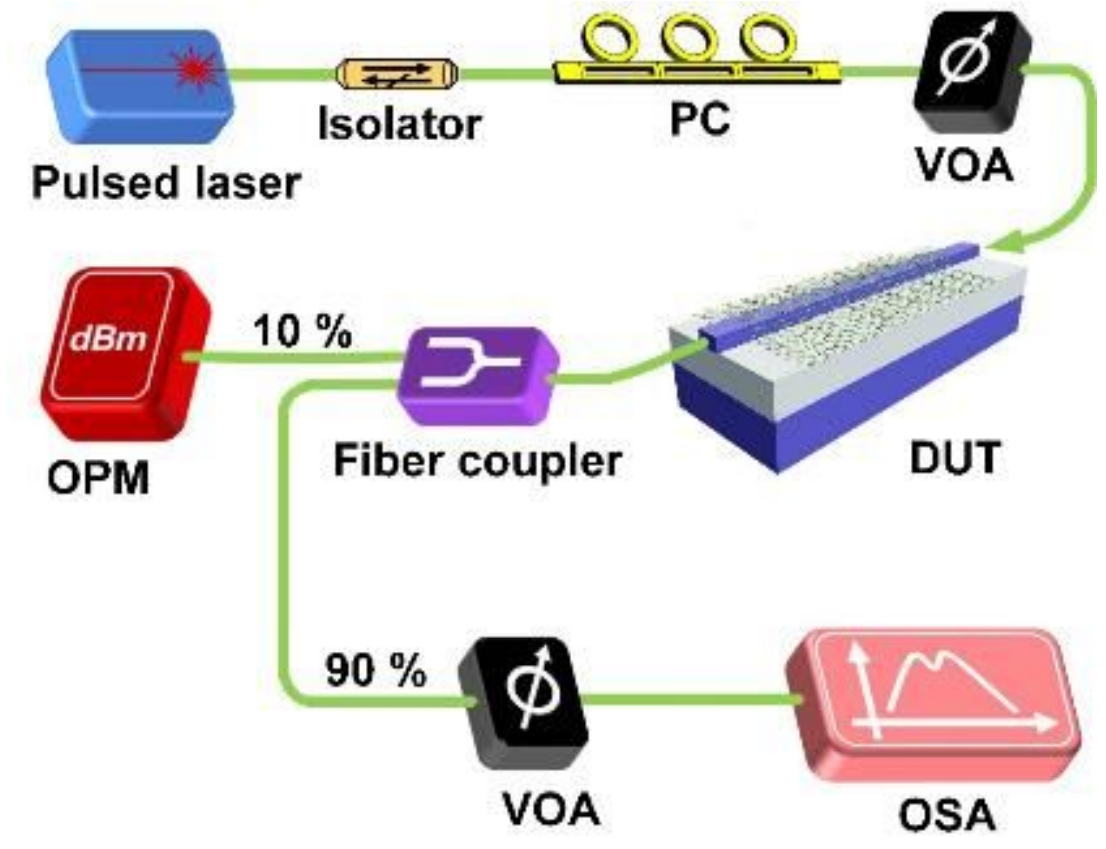

Figure 5

Experimental setup for SPM measurement in GO-coated SOI nanowires. PC: polarization controller. VOA: variable optical attenuator. OPM: optical power meter. DUT: device under test. OSA: optical spectrum analyzer. 

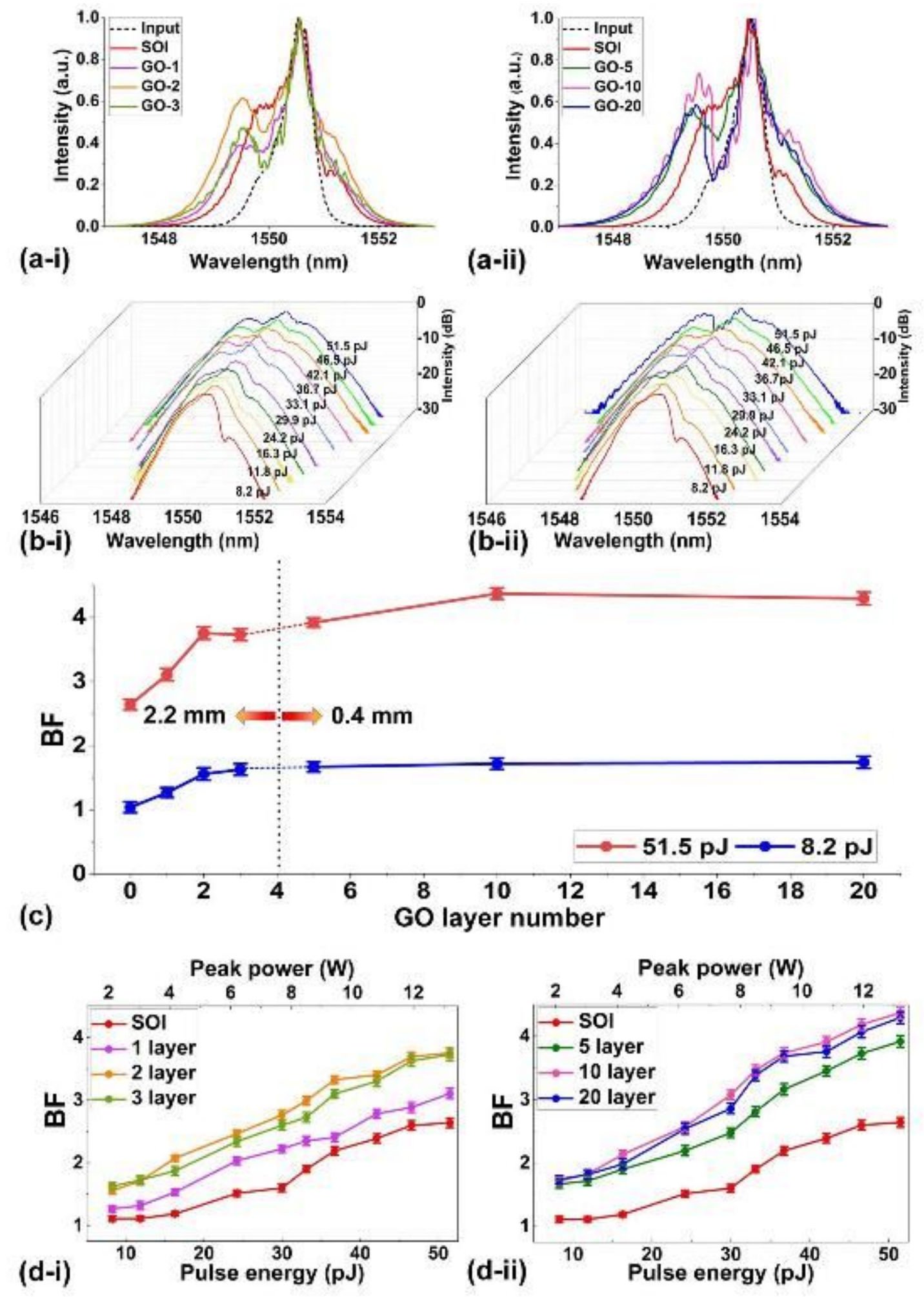

Figure 6

SPM experimental results. (a) Normalized spectra of optical pulses before and after going through the GO-coated SOI nanowires at a coupled pulse energy of $\sim 51.5 \mathrm{pJ}$. (b) Optical spectra measured at different pulse energies for the GO-coated SOI nanowires. (c) BFs of the measured output spectra versus GO layer number at fixed coupled pulse energies of $8.2 \mathrm{pJ}$ and $51.5 \mathrm{pJ}$. (d) BFs of the measured output spectra versus coupled pulse energy (or coupled peak power). In (a), (b) and (d), (i) and (ii) show the 
results for the SOI nanowires with 2.2-mm-long, 1-3 layers of GO and with 0.4-mm-long, 5-20 layers of GO, respectively. In (a), (c) and (d), the corresponding results for the bare SOI nanowires are also shown for comparison.

Peak power (W)
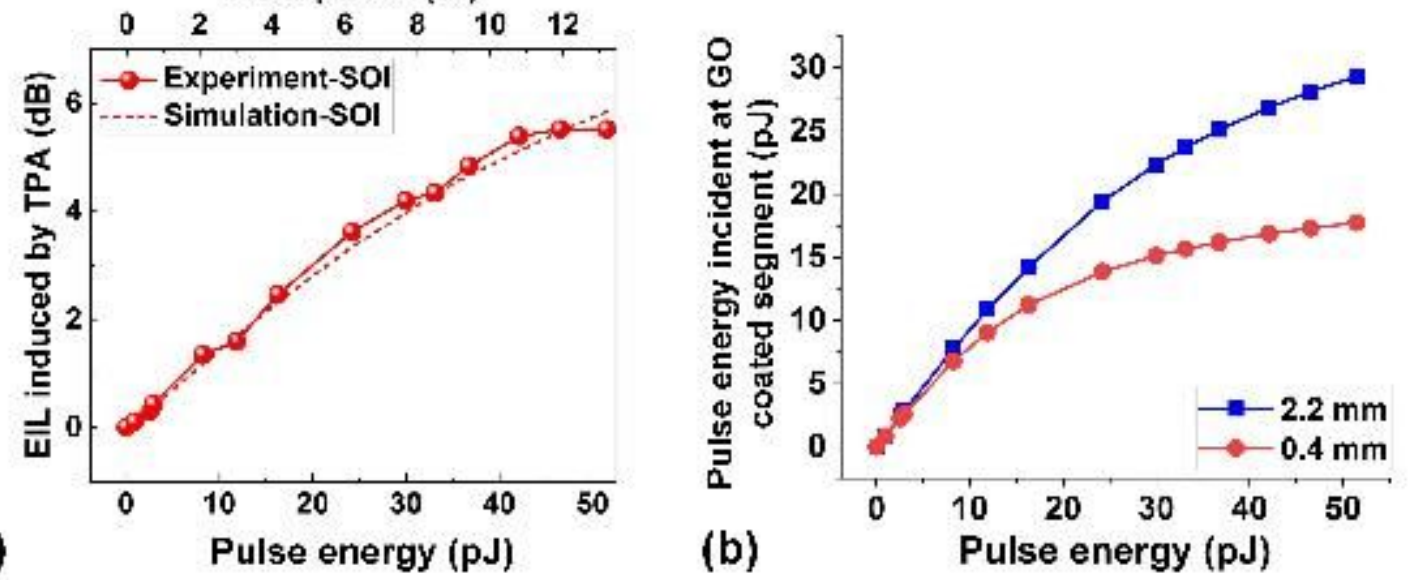

(b)

Pulse energy (pJ)

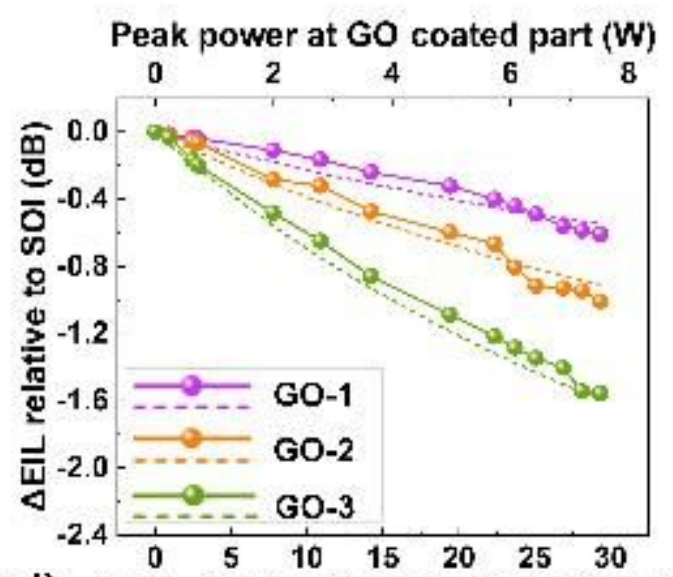

(C-i) Pulse energy at GO coated part (pJ)

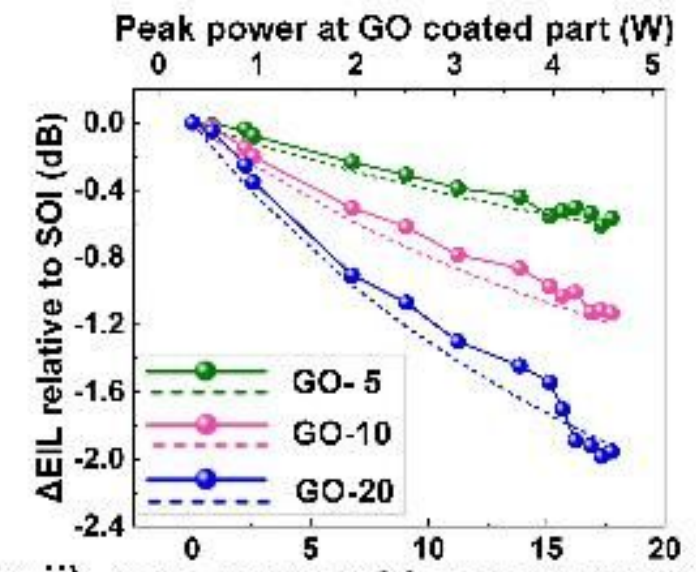

(c-ii) Pulse energy at GO coated part (pJ)

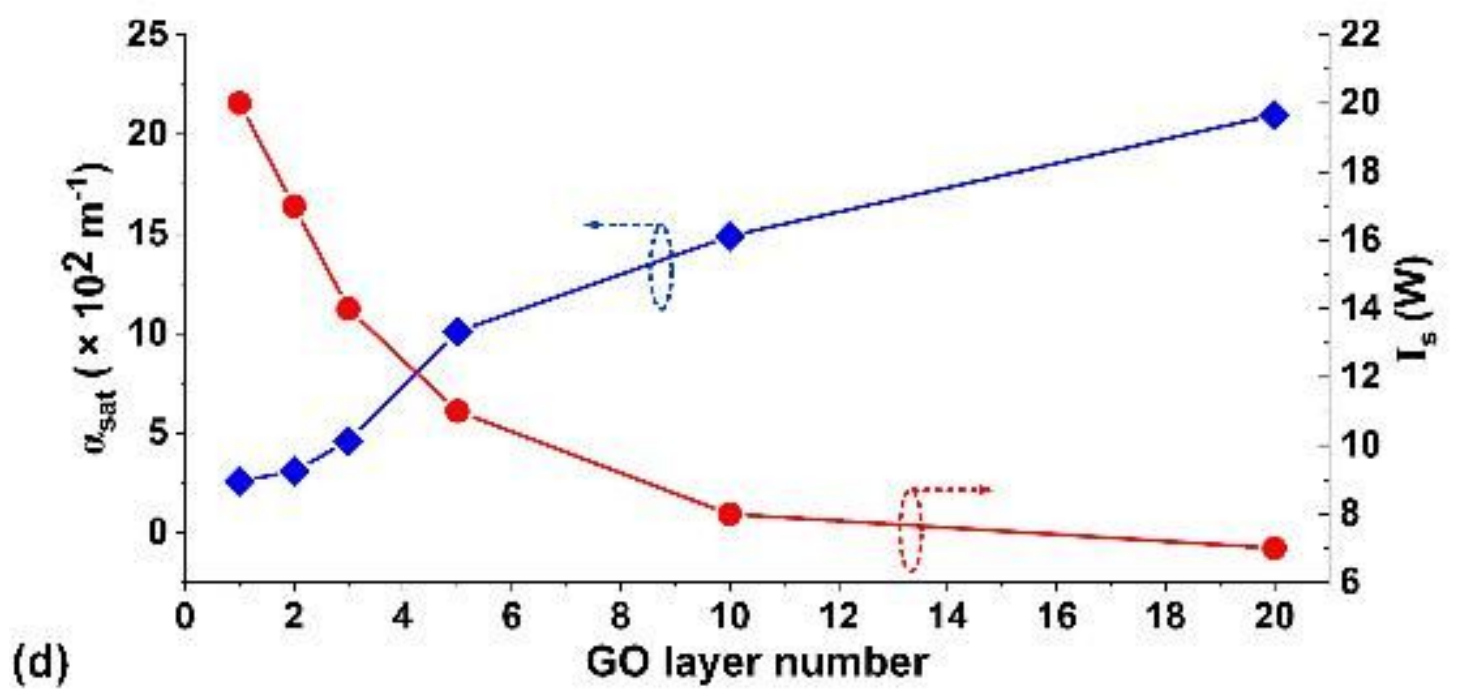

Figure 7

(a) Measured and simulated EIL induced by TPA and FCA of silicon. (b) Pulse energy incident at the 2.2mm-long and 0.4-mm-long GO coated segments versus pulse energy coupled into the hybrid waveguides. 
(c) Measured (solid curves) and fit (dashed curves) $\Delta$ EIL relative to SOI versus pulse energy incident at (i) 2.2-mm-long and (ii) 0.4-mm-long GO coated segments. (d) Fit asat and Is versus GO layer number.
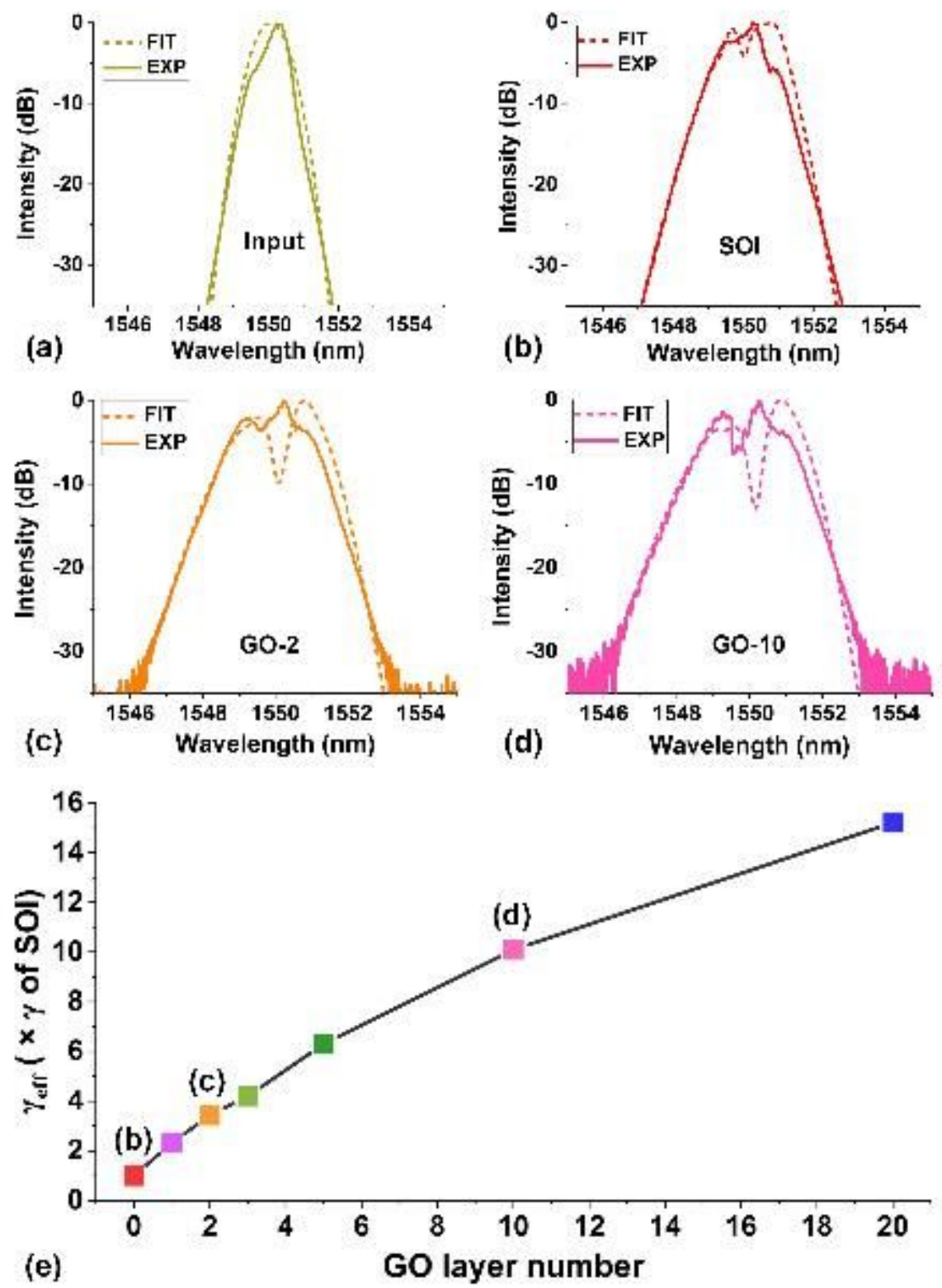

Figure 8

(a) -(d) Measured and fit optical spectra for (a) input optical pulses, (b) output signal after transmission through the bare SOI nanowire, (c) output signal after transmission through the SOI nanowire with 2.2mm-long, 2 layers of GO, and (d) output signal after transmission through the SOI nanowire with 0.4-mmlong, 10 layers of GO. (e) yeff of hybrid waveguides (normalized to y of the bare SOI nanowire) versus GO layer number. The data points corresponding to (b)-(d) are labelled in (e). The coupled pulse energy in (b) $-(\mathrm{e})$ is $\sim 51.5 \mathrm{pJ}$. 

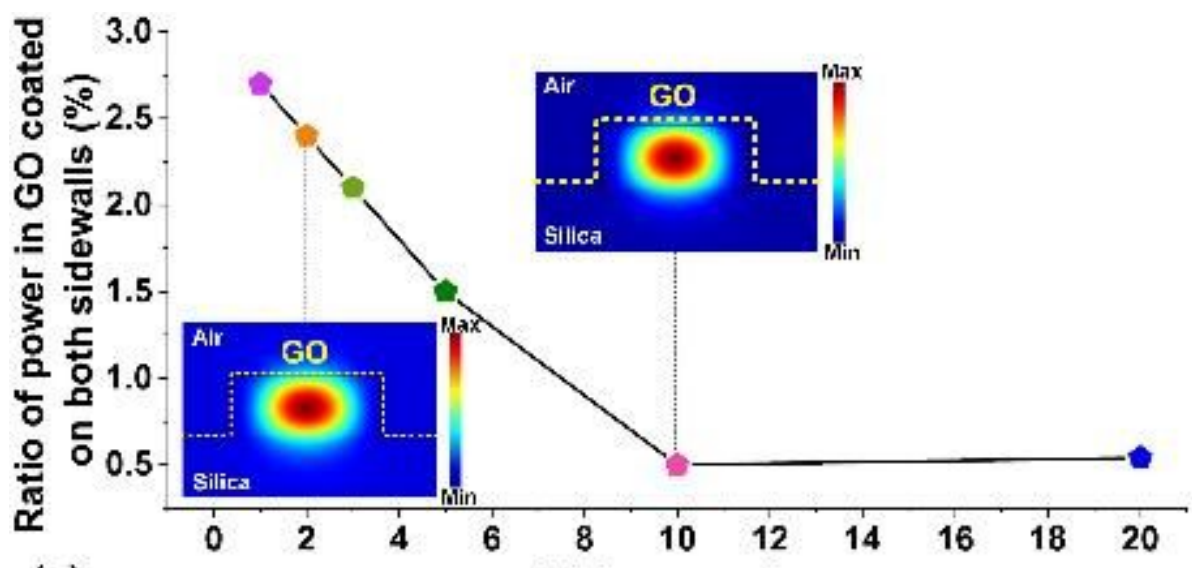

(a)

GO layer number

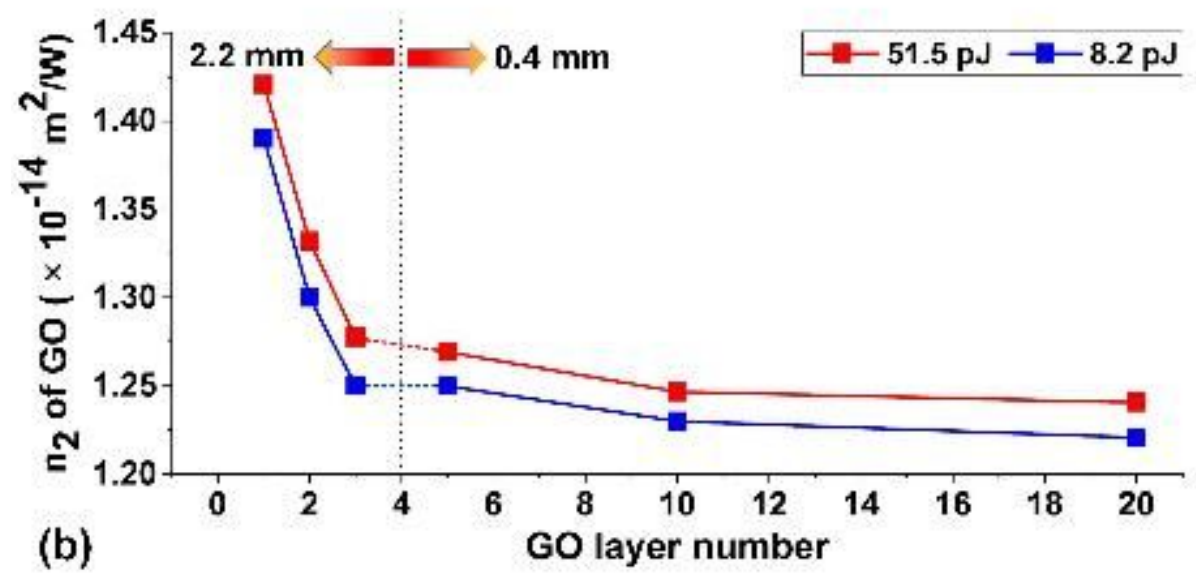

Peak power $(W)$

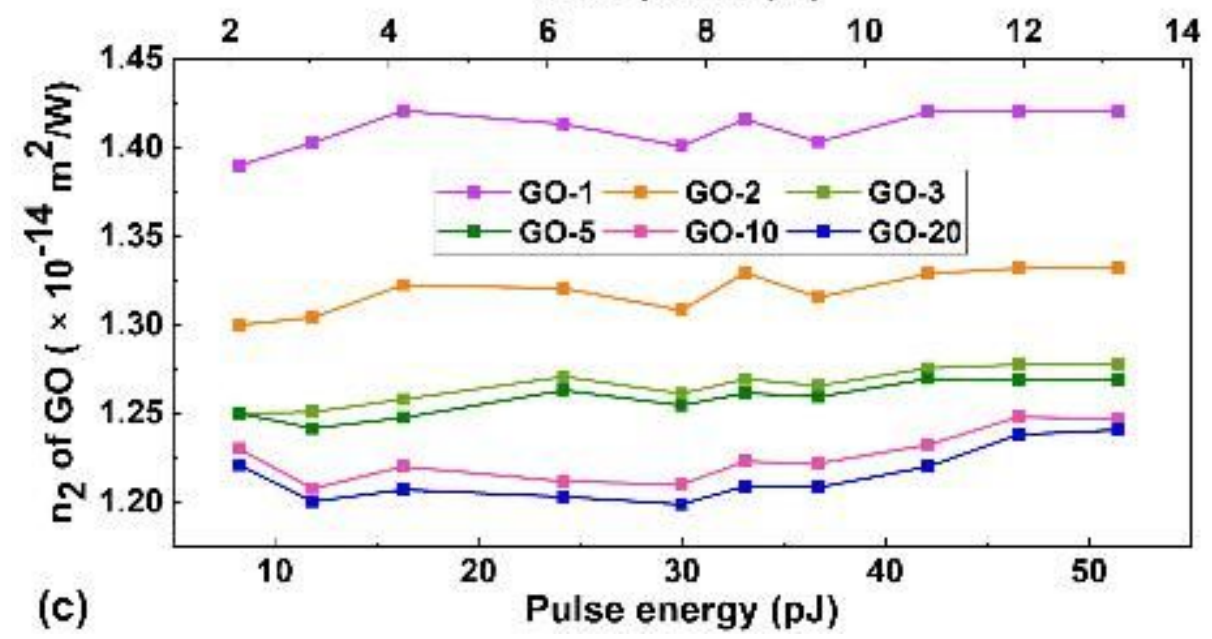

Figure 9

(a) Ratio of power in GO coated on both sidewalls to that in all GO material regions. Insets show the TE mode profiles for the SOI nanowires with 2 and 10 layers of GO, respectively. (b) n2 of GO versus layer number at fixed coupled pulse energies of $8.2 \mathrm{pJ}$ and $51.5 \mathrm{pJ}$. (c) n2 of $\mathrm{GO}$ versus coupled pulse energy (or coupled peak power) for the SOI nanowires with (i) 2.2-mm-long, 1-3 layers of GO and (ii) 0.4-mmlong, 5-20 layers of GO. 


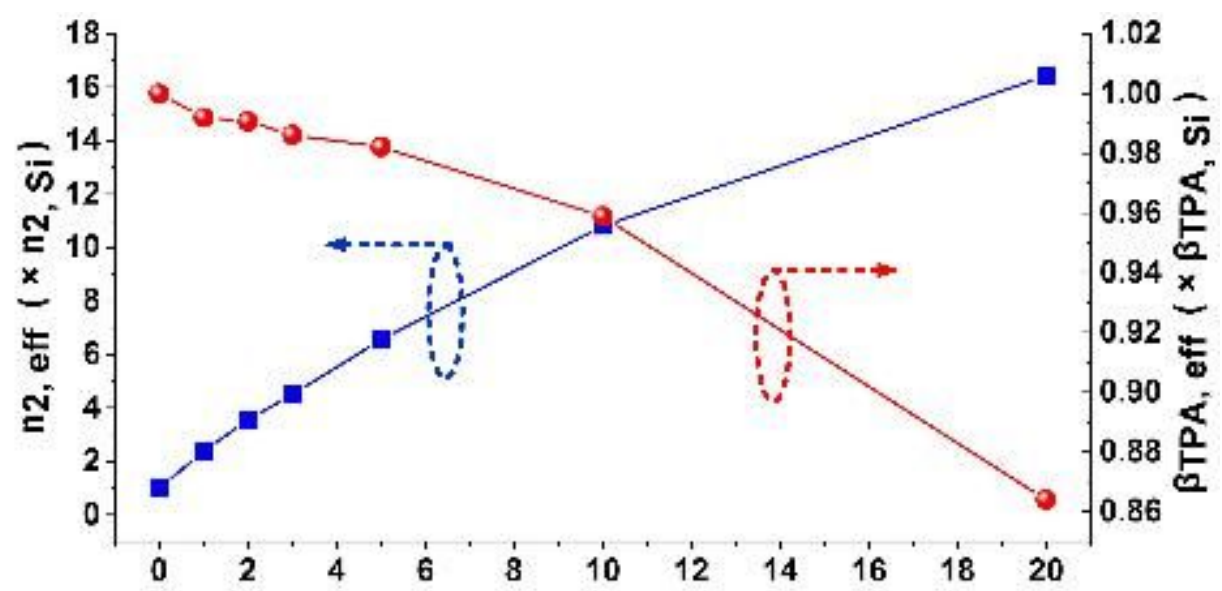

(a) GO layer number

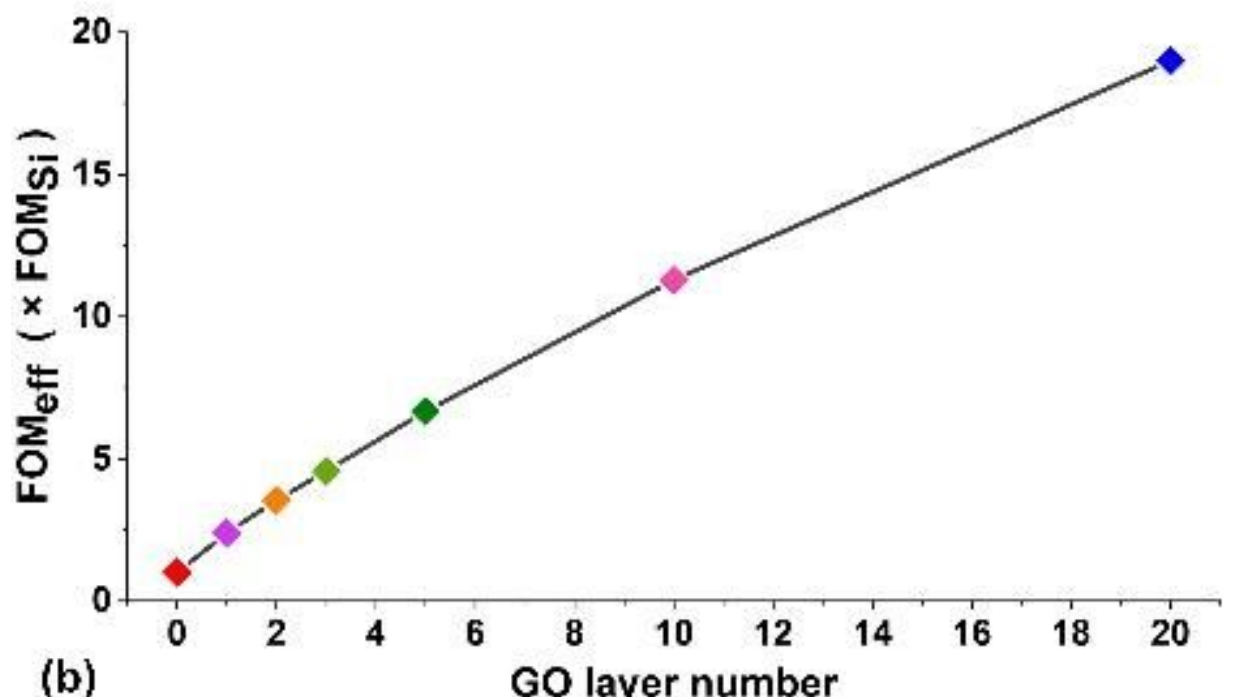

Figure 10

(a) n2, eff and $\beta T P A$, eff of the hybrid waveguides versus GO layer number. (b) FOMeff of the hybrid waveguides versus GO layer number. The coupled pulse energy in (a)-(b) is $~ 51.5 \mathrm{pJ}$. In (a) and (b), the corresponding results for silicon (GO layer number $=0$ ) are also shown for comparison. 